\title{
Andersen-Tawil Syndrome: A Retrospective Analysis of Clinical and Electrocardiographic Characteristics
}

\author{
SAHIL KOPPIKAR, MD ${ }^{1}$, HYUN YOO, MD ${ }^{2}$, ISABEL KONOPKA, MD ${ }^{2}$, NORMA PIZZARELLI, MD ${ }^{2}$, \\ ADRIAN BARANCHUK, MD, FACC FRCPC ${ }^{3}$ and RAFAEL ACUNZO, MD ${ }^{2}$ \\ ${ }^{1}$ Department of Internal Medicine, Kingston General Hospital, Queen's University, Kingston, Ontario, Canada \\ ${ }^{2}$ Division of Cardiology, Ramos Mejia Hospital, Buenos Aires, Argentina \\ ${ }^{3}$ Heart Rhythm Service, Kingston General Hospital, Queen's University, Kingston, Ontario, Canada
}

\begin{abstract}
Andersen-Tawil syndrome is an autosomal dominant, multisystem disorder characterized by periodic paralysis, dysmorphic features and cardiac arrhythmias. Mutations in the KCNJ2 gene, which encodes potassium channels, have been identified in individuals with Andersen-Tawil syndrome. These individuals can present with typical electrocardiographic findings that can prognosticate their tendency to develop lethal arrhythmias. However, literature on cardiac and clinical findings in this population is scarce. We analyzed the clinical characteristics, electrocardiographic patterns and the risk of potentially malignant cardiac arrhythmias in seven subjects with Andersen-Tawil syndrome. All patients reported episodes of palpitations. Forty-two percent had syncope or presyncope and $28 \%$ had a history of periodic paralysis. A family history of sudden death was present in $42.8 \%$. Electrocardiogram analysis showed prominent $U$-waves in $57 \%$. The mean QTc and QUc intervals were $432.7 \pm 23.23 \mathrm{~ms}$ and $599.1 \pm 83.82 \mathrm{~ms}$, respectively. Exercise stress testing induced ventricular arrhythmias in all patients in the initial stages. The 24-h Holter monitoring showed frequent ventricular premature beats (85\%) and episodes of nonsustained polymorphic or bidirectional ventricular tachycardia (71\%). Patients with AndersenTawil syndrome have a significant clinical burden of ventricular arrhythmias that can progress to sudden cardiac death. The 24-h Holter monitoring and exercise stress test were highly efficient methods to detect the presence of potentially lethal ventricular arrhythmias and should be used in the diagnostic work-up of patients with Andersen-Tawil syndrome.
\end{abstract}

KEYWORDS. Andersen-Tawil syndrome, electrocardiographic interpretation, genetic disease, sudden cardiac death, ventricular arrhythmias.
ISSN 2156-3977 (print) ISSN 2156-3993 (online)

(C) 2015 Innovations in Cardiac Rhythm Management

\section{Introduction}

Andersen-Tawil syndrome (ATS) is an autosomal dominant, genetic or sporadic, multisystem disorder characterized by developmental, cardiac, and neuromuscular abnormalities. ${ }^{1}$

The authors report no conflicts of interest for the published content. Manuscript received October 29, 2015, Final version accepted November 19, 2015.

Address correspondence to: Adrian Baranchuk, MD, FACC FRCPC, Associate Professor of Medicine, Cardiac Electrophysiology and Pacing, Kingston General Hospital K7L 2V7, Queen's University, Ontario, Canada. E-mail: barancha@kgh.kari.net
Although the first cases were reported in 1971 by Andersen and colleagues, the triad of cardinal clinical features (periodic paralysis, cardiac arrhythmias, and dysmorphic features) was first universally recognized in the $1990 \mathrm{~s}^{2-5}$

Mutations in the KCNI2 gene, which encodes the $\alpha$-subunit of the potassium channel Kir2.1, have been identified in patients with ATS. ${ }^{6}$ Currently, more than 20 mutations have been reported in the literature. ${ }^{7-12}$ The Kir2.1 potassium channel is responsible for the inward potassium rectifier current (IK1) during diastole. ${ }^{13-15}$ Disturbances during this phase of repolarization can result in significant arrhythmogenesis and potentially symptomatic 
or life-threatening rhythms. However, KCNJ2 mutations are detectable in only $60 \%$ of patients with ATS making genetic screening limited as a universal diagnostic tool. ${ }^{10}$ The diagnosis of ATS remains elusive and sometimes difficult to distinguish from other primary electrical disorders. ${ }^{6}$

Given the paucity of data in the literature, further studies are required to identify the clinical characteristics, electrocardiographic patterns, and risk of potentially malignant arrhythmias in ATS. In order to better define these distinctive features of ATS and enable earlier diagnosis, we conducted a retrospective standardized evaluation of seven subjects with clinically diagnosed ATS.

\section{Materials and methods}

\section{Study population}

Seven patients with ATS from a single academic center in Buenos Aires, Argentina, were included in the study. The clinical diagnosis of ATS was performed by the presence of at least two of the following criteria: 1) familial periodic paralysis; 2) characteristic facies, dental abnormalities, or small hands/feet, and at least two of low-set ears, widely spaced eyes, small mandible, fifth-digit clinodactyly, syndactyly; and 3) symptomatic cardiac arrhythmias or evidence from electrocardiograms (ECGs) of enlarged U-waves, ventricular ectopy, and prolonged QTc or QUc interval. Medical records of the patients were used to obtain demographics, and medical and treatment history. Patients were excluded from the study if they had any other known conditions that could cause the aforementioned ECG abnormalities or did not get a baseline ECG, exercise stress test and 24-h Holter monitoring. The study design was approved by the Ethics and Research Board at the Ramos Mejia Hospital in Argentina. All patients provided informed consent to participate in the study.

\section{Electrocardiographic analysis}

A 12-lead electrocardiogram (DM CARDIOSCAN Resting 12 Lead ECG 4.0, Beijing, China) at a paper speed of $25 \mathrm{~mm} / \mathrm{s}$ was recorded at rest in the supine position during the patient's first presentation prior to treatment. The RR, QT, and QU intervals were compared in all the patients. The $\mathrm{T}$ and $\mathrm{U}$-waves were measured in the lead with the highest amplitude, usually in V2 or V3. The QT interval was measured from the onset of QRS to the end of the T-wave, at the point at which the T-wave downslope crossed the isoelectric line. ${ }^{16}$ The $\mathrm{U}$-wave was defined as an early diastolic deflection after the end of the T-wave. ${ }^{17}$ The QU interval (onset of QRS to the end of the U-wave) was also measured. The corrected QT and QU intervals (QTc and QUc) were calculated using Bazett's formula and averaged from three consecutive sinus beats. The results were analyzed and calculated by two separate investigators.

\section{Exercise stress test}

All the patients underwent a uniform exercise protocol using a cycle ergometer. The initial phase consisted of
3 min of pedaling in an unloaded state followed by a ramp protocol involving an increasing work rate to maximal exercise. This was also conducted once during initial investigation prior to treatment.

\section{4-h Holter monitoring}

Holter monitor studies were performed for $24 \mathrm{~h}$ with a three-channel device (DM CARDIOSCAN II Premier 12.0). Similar to other investigations, the Holter monitoring was conducted once prior to treatment.

\section{Treatment and follow-up}

All patients were treated as per current standard of care by their clinician. All patients were followed up regularly as per their clinician's judgment. Patients' symptoms, medications, and any changes were recorded at each visit.

\section{Results}

\section{Clinical characteristics}

The average age at enrolment was 21 years. Four participants were female, the remaining three were male. All seven patients reported episodes of palpitations, while three of them associated it with presyncope or syncope $(42.8 \%)$. Two subjects $(28.5 \%)$ had a history of periodic paralysis. A family history of sudden death was present in three subjects $(42.8 \%)$. All participants in the study had dysmorphic features such as short stature and micrognathia (Table 1).

\section{Baseline ECG analysis}

Baseline ECGs showed prominent U-waves in four subjects (Table 2, Figure 1). Ventricular bigeminy, polymorphic ventricular premature beats and bidirectional ventricular tachycardia was also observed in three separate participants (Table 2, Figure 2). The mean QTc interval was 432.7 $\pm 23.23 \mathrm{~ms}$. However, the QUc interval was significantly prolonged at $599.1 \pm 83.82 \mathrm{~ms}$ (Table 2).

\section{Exercise stress test}

Exercise stress testing revealed premature ventricular beats in all seven subjects that resolved during peak

Table 1: Clinical characteristics of subjects in the study

\begin{tabular}{lc}
\hline Clinical characteristic & $\begin{array}{c}\text { Number of subjects } \\
(\%)\end{array}$ \\
\hline Age & $21 \pm 14$ (years) \\
Female & $4(57)$ \\
Palpitations & $7(100)$ \\
Syncope or presyncope & $3(42.8)$ \\
Family history of sudden cardiac & $3(42.8)$ \\
$\quad$ death & $2(28.5)$ \\
History of periodic paralysis & $7(100)$ \\
Dysmorphic features & \\
\hline
\end{tabular}


Table 2: ECG characteristics, exercise stress testing, Holter monitoring and EPS results in seven subjects

\begin{tabular}{lc}
\hline Baseline ECGs & $\begin{array}{c}\text { Number of } \\
\text { subjects (\%) }\end{array}$ \\
\hline $\begin{array}{l}\text { Prominent U waves } \\
\text { Ventricular bigeminy }\end{array}$ & $4(57.1 \%)$ \\
Polymorphic ventricular premature beats & $1(14.2 \%)$ \\
Bidirectional ventricular tachycardia & $1(14.2 \%)$ \\
QTc intervals & $1(14.2 \%)$ \\
QUc intervals & $432.7 \pm 23.23 \mathrm{~ms}$ \\
Exercise stress testing & $599.1 \pm 83.82 \mathrm{~ms}$ \\
$\begin{array}{l}\text { Premature ventricular beats } \\
\text { Bidirectional or polymorphic ventricular }\end{array}$ & $1(14.2 \%)$ \\
$\quad$ tachycardia & \\
24-h Holter monitoring & \\
$\begin{array}{l}\text { Premature ventricular beats } \\
\text { Bidirectional or polymorphic ventricular } \\
\quad \text { tachycardia }\end{array}$ & $6(85.7 \%)$ \\
$\begin{array}{l}\text { Electrophysiological study } \\
\text { Ventricular tachycardia/ventricular } \\
\quad \text { fibrillation }\end{array}$ & \\
\hline
\end{tabular}

exercise (Table 2, Figure 3). One of those participants developed episodes of polymorphic ventricular tachycardia at the beginning of the stress test, which was attenuated with increasing effort (Figure 4). This patient had prominent $U$ waves on a baseline ECG.

\section{4-h Holter monitoring}

Frequent premature ventricular beats (isolated, bigeminy, and couplet) were observed in six of the subjects (85\%). The records of five patients (71\%) showed episodes of nonsustained polymorphic or bidirectional ventricular tachycardia (Table 2, Figure 5).

\section{Electrophysiological study}

If the Holter showed non-sustained polymorphic or bidirectional ventricular arrhythmias, electrophysiological study (EPS) was performed to check for inducibility. Four of the patients underwent EPS. The induction of tachyarrhythmia was negative in three patients. One patient developed a polymorphic ventricular tachycardia (VT) that degenerated into ventricular fibrillation (VF) requiring electrical cardioversion and insertion of an implantable cardioverter-defibrillator (ICD) (Table 2). An ICD was recommended in our patients when polymorphic VT/VF was easily induced with a non-aggressive protocol.

\section{Follow-up}

Four patients were treated with nadolol (57\%). Atenolol and diltiazem were used in one patient each. One patient received amiodarone prior to the diagnosis of ATS, but was changed to propranolol upon diagnosing ATS. Two patients received spironolactone due to recurrent episodes of hypokalemia associated with $\beta$-blocker use (Table 3). During a follow-up of $105 \pm 135$ months, all patients remained asymptomatic.
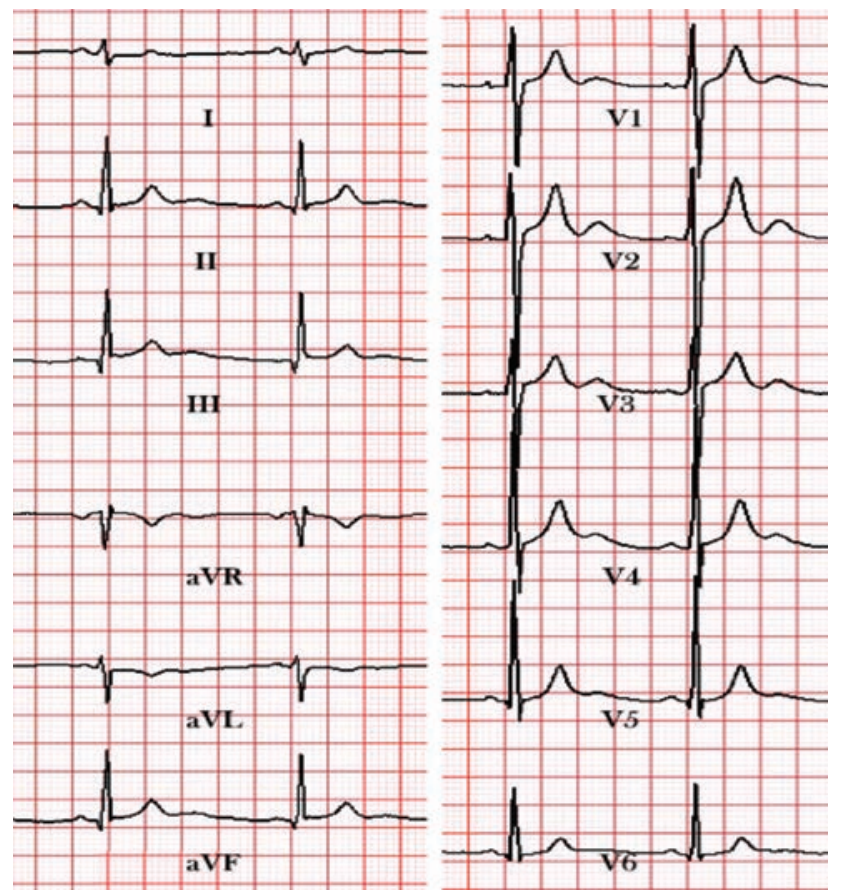

Figure 1: Baseline electrocardiogram of patient 1 with the presence of prominent $\mathrm{U}$ waves in $\mathrm{V} 1-\mathrm{V} 4$ at rest.

\section{Discussion}

ATS is a heterogeneous autosomal dominant disorder characterized by the presence of muscular periodic paralysis, developmental abnormalities, and cardiac arrhythmias. The full triad of clinical features has been reported in up to $78 \%$ of mutation-positive patients in previous studies. ${ }^{1}$ Because of this, a clinical diagnosis of ATS has traditionally been made in individuals with two of the three cardinal features. However, many of these patients present with typical cardiac abnormalities. Sixty percent of patients with ATS present mutations of the KCNJ2 gene located on chromosome 17 (17q23), which provides the code for the synthesis of the rectifying potassium channel Kir2.1 activated during cardiac repolarization. ${ }^{7,8,10,11}$ The Kir 2.1 potassium channel is responsible for the IK1. In the cardiac myocytes, IK1 plays an important role in stabilizing resting potential and determining the shape of the terminal portion of the cardiac action potential. During the terminal phase of repolarization and during diastole, IK1 dominates membrane conductance and modulates cell excitability. ${ }^{13-15}$ Disturbances during this phase of repolarization can result in significant arrhythmogenesis and potentially symptomatic or life-threatening rhythms.

Several electrocardiographic abnormalities in patients with ATS have been described previously. Zhang et al. ${ }^{18}$ described prominent U-waves and prolonged QT-U interval compared with healthy individuals $(650 \mathrm{~ms}$ versus $600 \mathrm{~ms})$. They showed an abnormal T-U wave in $91 \%$ of their patients. These abnormalities included:

1. prolonged terminal portion of the descending $\mathrm{T}$-wave $(70 \%)$

2. wide $\mathrm{T}-\mathrm{U}$ wave junction $(43 \%)$

3. biphasic U waves (16\%)

4. large U waves $(73 \%)$. 


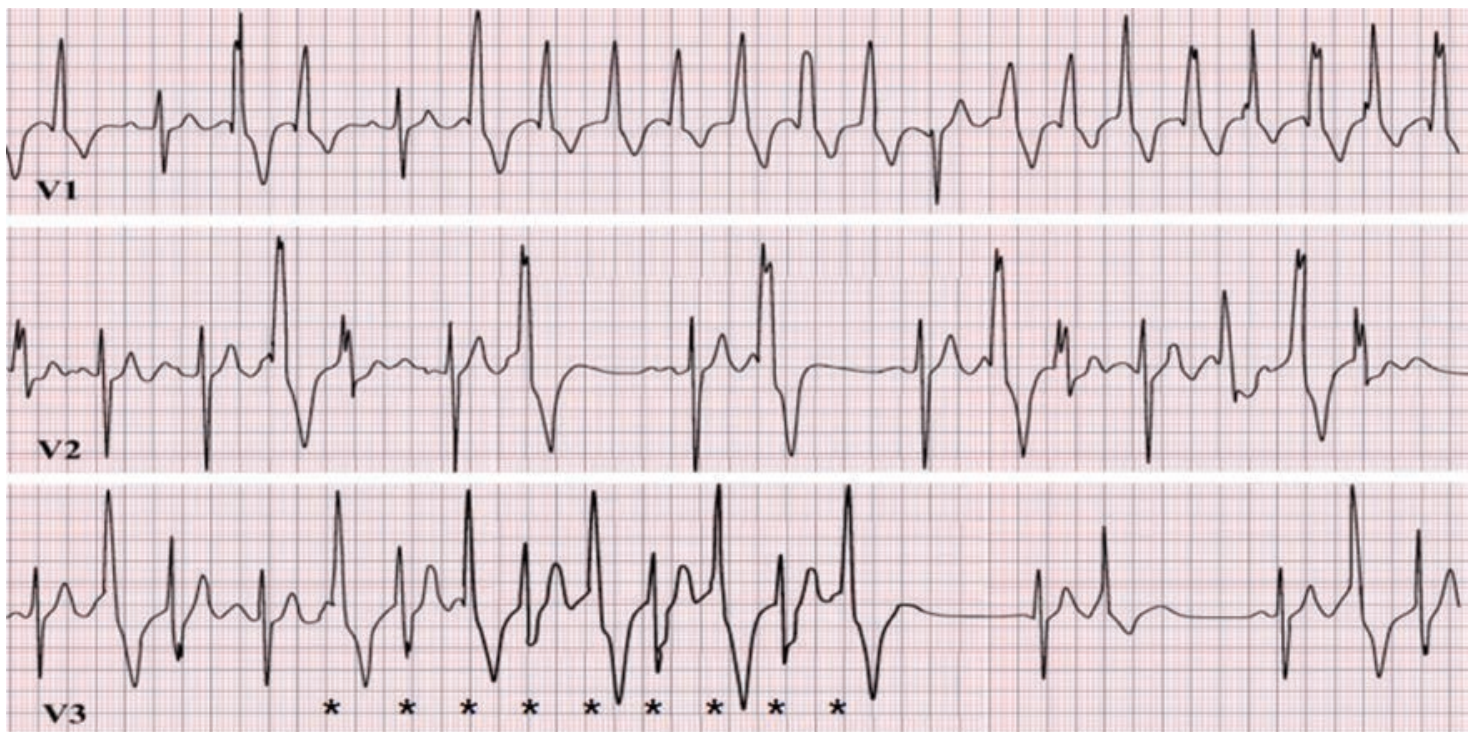

Figure 2: Baseline electrocardiogram of patient 1 showing the spontaneous development of bidirectional ventricular tachycardia (black asterisk). This dysrhythmia is characterized by two distinct QRS morphologies alternating every other beat. Both morphologies meet criteria for a ventricular origin.

Recently, Kukla et al. ${ }^{6}$ have proposed five new electrocardiographic criteria for the diagnosis of ATS: 1) "U on $\mathrm{P}^{\text {" }}$ sign (U-wave masquerading $\mathrm{P}$-wave) during sinus tachycardia, 2) pseudo "Tee-pee sign" during a premature ventricular contraction by prolongation of the descending limb of the T+U-wave, 3) post- extrasystolic "pseudo-LQTSpattern" that mimics long-QT syndrome (LQTS), 4) the presence of U-waves in the inferior leads and precordial leads V2-V3, 5) and U-wave amplitude augmentation after adrenaline administration. ${ }^{6}$

In our study, four patients (57.14\%) had prominent U-waves on ECG recording (Figure 1). This percentage was slightly lower than, but similar to, previous studies. ${ }^{18}$ Furthermore, the QUc interval was relatively shorter than previously reported (599.1 ms versus $650 \mathrm{~ms}$ ). The differences between studies can be explained by small sample sizes and slightly differing definitions of large U-wave measurements. All four patients with large U-waves demonstrated the "U on P sign" during sinus tachycardia induced by exercise testing. Kukla et $\mathrm{al}^{6}{ }^{6}$ were able to observe this sign in five out of their six patients. The pseudo "Tee-pee sign," the post-extrasystolic "pseudo-LQTS-pattern" and the presence of U-waves in the inferior leads were observed in two patients each. Previous studies have proposed ATS as LQT7 on the basis of an apparently long QTc interval and associated arrhythmias. ${ }^{19}$ These studies may have reported a more pronounced QTc prolongation on the basis of including the U-wave in the QT measurement. The degree of a true QTc prolongation in our patients was very modest with a mean QTc interval of $432.7 \pm 23.23 \mathrm{~ms}$. The electrocardiographic changes suggested in previous studies were consistently found in our population. Accounting for the small sample size, prominent Uwaves and the "U on P sign" seem to be the most consistent across studies. It should be noted that ventricular arrhythmias were part of diagnostic criteria for ATS in this study, and, hence, could be contributing to a high prevalence in our study. These findings suggest that electrocardiographic changes may not be particularly useful in diagnosing ATS, but when present can be highly specific for ATS and prognosticate potentially lethal arrhythmias in those patients. However, further larger studies are needed in this area to validate the aforementioned electrocardiographic criteria.

Patients affected by this syndrome have a greater tendency to develop ventricular arrhythmias, consisting of premature ventricular contractions (isolated, bigeminy, and couplet) and non-sustained polymorphic or bidirectional ventricular tachycardia. ${ }^{1}$ However, in the literature, the incidence of torsade de pointes, cardiac arrest, and sudden cardiac death is rare. ${ }^{10,12,19}$ Interestingly, given the significant tachycardia burden from ventricular ectopy in many patients, a large majority remain asymptomatic. ${ }^{20}$ However, in our study, all patients were symptomatic with palpitations and $42.8 \%$ of our subjects had a family history of sudden cardiac death. Premature ventricular beats and bidirectional or polymorphic ventricular tachycardia was identified in a majority of our subjects (Table 2). Bidirectional ventricular tachycardia is an extremely rare form of ectopy described in only three clinical settings: ATS, digitalis toxicity, and catecholaminergic ventricular tachycardia. Bidirectional ventricular tachycardia can progress to ventricular fibrillation and death. These arrhythmias attenuated during peak exercise in our patients with ATS, a characteristic that differs from catecholaminergic ventricular tachycardia. This phenomenon has been previously observed in the literature. ${ }^{6}$ According to our findings, 24-h Holter monitors and exercise stress testing were highly efficient methods to detect the presence of potentially lethal ventricular arrhythmias. Given the potential for sudden cardiac death and the efficacy of testing in these patients, we recommend both 


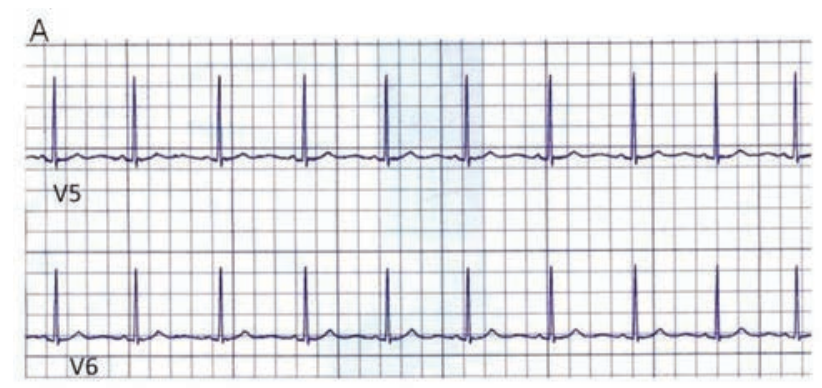

B
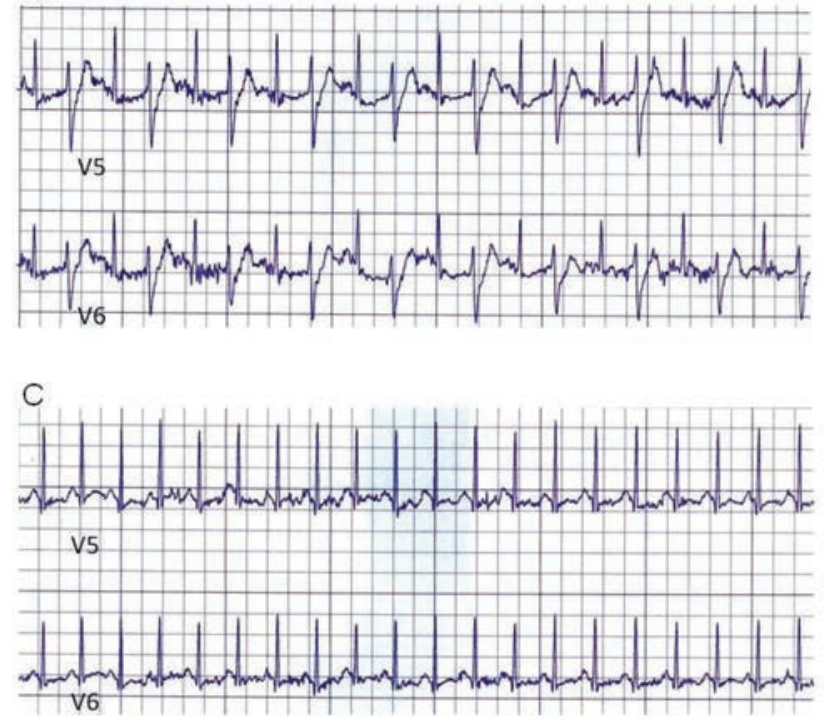

Figure 3: Electrocardiogram (ECG) monitoring of Patient 3 during exercise stress testing. (A) Baseline ECG just prior to exercise showing normal sinus rhythm. (B) Frequent premature ventricular beats are noted in the initial stages. (C) The resolution of arrhythmias during peak exercise with sinus tachycardia. This pattern was documented in all patients in the study.

a 12-lead ECG and a 24-h Holter monitoring as standard tests in the diagnostic work-up of individuals with suspected ATS. The management of patients with ATS is currently empirical and individualized. There still is no consensus on how to treat these patients. While medical therapy for polymorphic ventricular tachycardia has been minimally successful, $\beta$-blockers and calcium-channel blockers seem to be the most efficacious and commonly used., ${ }^{32,21}$ Most of our patients were on a $\beta$-blocker with good effect (Table 3). More recently, flecainide has been shown to be efficacious and safe in suppressing ventricular arrhythmias in patients with ATS. ${ }^{23,24}$ Importantly, clinical conditions that might trigger hypokalemia such as diarrhea or medications that could prolong the QT interval should be avoided. Potassium replacement should be promptly initiated and potassiumsparing medications, such as spironolactone, should be used when needed.

In general, genetic forms of ventricular ectopy and tachyarrhythmias have been difficult to induce in electrophysiological studies and have not been amenable to ablation. $3,8,12$
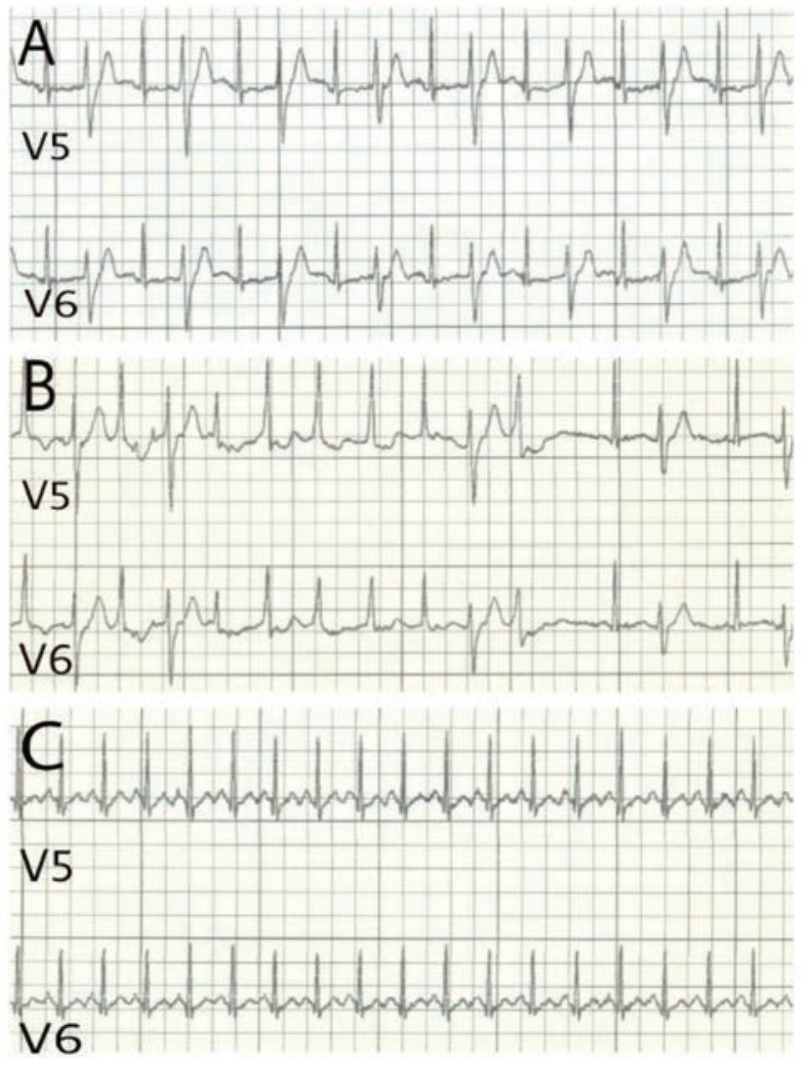

Figure 4: Electrocardiogram monitoring of Patient 1 during exercise stress testing. (A) Premature ventricular beats and (B) polymorphic ventricular tachycardia in the initial stages of the stress test are noted. (C) These arrhythmias attenuated with increasing effort at peak exercise.

Most reports in the field have been case series, and no consensus for risk stratification or treatment indications have been established. The efficacy of an ICD for unstable ventricular tachycardia or ventricular fibrillation has been well characterized; however, the indications for ICD use in inherited arrhythmia syndromes remains unclear. Currently, a family history of an inheritable arrhythmogenic condition is considered a class Ilb indication for ICD implantation as per the 2012 AHA guidelines; however, the presence of spontaneous sustained ventricular tachycardia is considered a class I indication for device placement. ${ }^{22}$ In our patients, we extrapolated the approach from other inherited arrhythmia disorders. If the Holter showed non-sustained polymorphic or bidirectional ventricular arrhythmias, EPS was performed to check for inducibility. An ICD was recommended when polymorphic VT/VF was easily induced with a non-aggressive protocol. Contraindications to ICD placement include disorders that are amenable to medical treatment of radiofrequency ablation; however, as mentioned earlier, many forms of ATS arrhythmias demonstrate resistance to such forms of therapy. ${ }^{12}$ Given the lack of clear guidelines for treating these patients, therapy should be individualized and tailored to the patient's findings on clinical, ECG, and Holter examinations. 


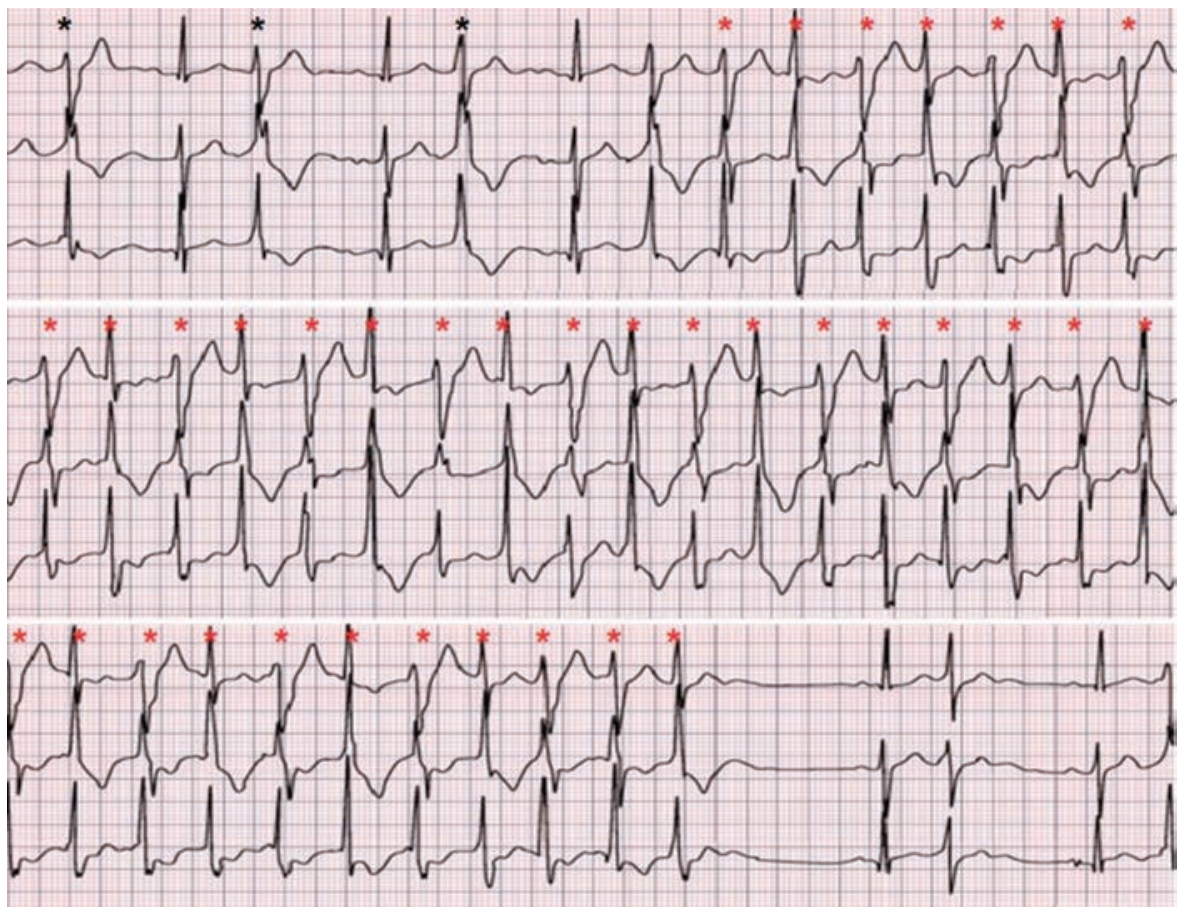

Figure 5: 24-h Holter monitoring record for Patient 1. The recording shows spontaneous occurrence of premature ventricular beats (black asterisk) and runs of non-sustained bidirectional ventricular tachycardia.

Table 3: Treatment modality used in all subjects from enrolment to end of study

\begin{tabular}{lc}
\hline Treatment & Number of subjects (\%) \\
\hline Nadolol & $4(57.1)$ \\
Spironolactone & $2(28.5)$ \\
Atenolol & $1(14.2)$ \\
Diltiazem & $1(14.2)$ \\
Propranolol & $1(14.2)$ \\
Implantable cardioverter- $\quad 1(14.2)$ \\
$\quad$ defibrillator \\
\hline
\end{tabular}

\section{Limitations}

Because of the lack of availability in performing genetic testing at the subjects' centers, the diagnosis of ATS was based on clinical and electrocardiographic criteria.

\section{Conclusions}

Phenotypic and electrocardiographic heterogeneity limit the clinician's ability to identify patients with ATS who are at the highest risk for life-threatening arrhythmias. Our study, in addition to previous reports, continues to demonstrate a high prevalence of ventricular arrhythmias, some of which may progress to potentially malignant arrhythmias causing sudden cardiac death. ECG findings consistent in patients with ATS can be used to identify those at high risk. Exercise stress testing and 24-h Holter monitoring were highly efficient methods to detect the presence of these lethal arrhythmias.

\section{References}

1. Yoon G, Oberoi S, Tristani-Firouzi M, et al. Andersen-Tawil syndrome: Prospective cohort analysis and expansion of the phenotype. Am J Med Genet. 2006;140(4):312-321.

2. Andersen ED, Krasilnjkoff PA, Overvad H. Intermittent muscular weakness, extrasystoles, and multiple developmental anomalies: A new syndrome? Acta Padiatr Scand. 1971;60(5):559-564.

3. Tawil R, Ptacek LJ, Pavlakis SG, et al. Andersen's syndrome: Potassium-sensitive periodic paralysis, ventricular ectopy, and dysmorphic features. Ann Neurol. 1994;35(3):326-330.

4. Sansone V, Griggs RC, Meola G, et al. Andersen's syndrome: A distinct periodic paralysis. Ann Neurol. 1997;42 (3):305-312.

5. Canún S, Pérez N, Beirana LG. Andersen syndrome autosomal dominant in three generations. Am J Med Genet. 1999;85(2):147-156.

6. Kukla P, Biernacka K, Baranchuk A, et al. New Electrocardiographic Criteria for Diagnosis of Type-1 AndersenTawil Syndrome. Curr Cardiol Rev. 2014;10(3):222-228.

7. Plaster NM, Tawil R, Tristani-Firouzi M, et al. Mutations in Kir2.1 cause the developmental and episodic electrical phenotypes of Andersen's syndrome. Cell. 2001;105(4):511519.

8. Andelfinger G, Tapper AR, Welch RC, et al. KCNJ2 mutation results in Andersen syndrome with sex-specific cardiac and skeletal muscle phenotypes. Am J Hum Genet. 2002;71(3): 663-668.

9. Ai T, Fujiwara Y, Tsuji K, et al. Novel KCNJ2 mutation in familial periodic paralysis with ventricular dysrhythmia. Circulation. 2002;105(22):2592-2594.

10. Donaldson MR, Jensen JL, Tristani-Firouzi $M$, et al. PIP2 binding residues of Kir2.1 are common targets of mutations causing Andersen syndrome. Neurology. 2003;60(11):1811-1816. 
11. Bendahhou S, Donaldson MR, Plaster NM, Tristani-Firouzi M, Fu YH, Ptácek LJ. Defective potassium channel Kir2.1 trafficking underlies Andersen-Tawil syndrome. J Biol Chem. 2003;278(51):51779-51785.

12. Chun TUH, Epstein MR, Dick M, et al. Polymorphic ventricular tachycardia and KCNJ2 mutations. Heart Rhythm. 2004;1(2):235-241.

13. Lopatin AN, Nichols CG. Inward rectifiers in the heart: An update on I(K1). J Mol Cell Cardiol. 2001;33(4):625-638.

14. Wang Z, Yue L, White M, Pelletier G, Nattel S. Differential distribution of inward rectifier potassium channel transcripts in human atrium versus ventricle. Circulation. 1998;98(22): 2422-2428.

15. Liu GX, Derst C, Schlichthörl G, et al. Comparison of cloned Kir2 channels with native inward rectifier $\mathrm{K}^{+}$channels from guinea-pig cardiomyocytes. J Physiol. 2001;532(Pt 1):115-126.

16. Lepeschkin BE, Surawicz B. The measurement of the Q-T interval of the electrocardiogram. Circulation. 1952;6:378-388.

17. Surawicz B. U wave: Facts, hypotheses, misconceptions, and misnomers. J Cardiovasc Electrophysiol. 1998;9(10):1117-1128.

18. Zhang L, Benson DW, Tristani-Firouzi M, et al. Electrocardiographic features in Andersen-Tawil syndrome patients with KCNJ2 mutations: Characteristic T-U-wave patterns predict the KCNJ2 genotype. Circulation. 2005;111(21):2720-2726.
19. Tristani-Firouzi M, Jensen JL, Donaldson MR, et al. Functional and clinical characterization of KCNJ2 mutations associated with LQT7 (Andersen syndrome). J Clin Invest. 2002;110(3):381-388.

20. Schwartz PJ, Priori SG, Spazzolini C, et al. Genotype-Phenotype Correlation in the Long-QT Syndrome: gene-specific triggers for life-threatening arrhythmias. Circulation. 2001;103(1):89-95.

21. Katz JS, Wolfe GI, Iannaccone S, Bryan WW, Barohn RJ. The exercise test in Andersen syndrome. Arch Neurol. 1999;56 (3):352-356.

22. Tracy CM, Epstein AE, Darbar D, et al. 2012 ACCF/AHA/ HRS Focused Update Incorporated Into the ACCF/AHA/ HRS 2008 Guidelines for Device-Based Therapy of Cardiac Rhythm Abnormalities: A Report of the American College of Cardiology Foundation/American Heart Association Task Force on Practice Guide. J Am Coll Cardiol. 2013;61(3):e6-e75.

23. Miyamoto K, Aiba T, Kimura H, et al. Efficacy and safety of flecainide for ventricular arrhythmias in patients with Andersen-Tawil syndrome with KCNJ2 mutations. Heart Rhythm. 2015;12(3):596-603.

24. Fox D, Klein G, Hahn A, et al. Reduction of complex ventricular ectopy and improvement in exercise capacity with flecainide therapy in Andersen-Tawil syndrome. Europace. 2008;10(8):1006-1008. 